\title{
Scheduling coupled photovoltaic, battery and conventional energy sources to maximize profit using linear programming
}

\author{
David Torres, Jorge Crichigno, Gregg Padilla, Ruben Rivera \\ Northern New Mexico College 921 N. Paseo de Oñate, Española, NM 87532
}

\begin{abstract}
To address the increase of electricity demand, the need for reducing carbon dioxide, and the reduction of available fossil fuel resources, renewable energy sources are being recruited. Specifically energy generated by photovoltaic (PV) cells is becoming one of the most promising alternatives. In this context, this paper presents an optimization model for the scheduling problem where conventional and photovoltaic sources of energy are scheduled to be delivered to satisfy energy demand. The optimization model is formulated as a Linear Program (LP) with a bounded number of variables and constraints. The respective solution can be obtained in polynomial time and provides the optimal combination or schedule of energy generated from different sources (conventional, renewable and battery storage) such that the total demand is satisfied and the profit is maximized. Numerical results demonstrate the effectiveness and the generality of the scheme.
\end{abstract}

Keywords: Photovoltaic energy, Optimization, Scheduling, Linear Programming

\section{Introduction}

To face the increase of the electricity demand, the reduction of fossil fuel resources and the need for reducing carbon dioxide emissions, utility companies have turned to grid connected renewable power systems. PV generation is one of the most promising renewable sources of energy [1]. Moreover, recent advances in energy storage, which have been a barrier to realizing the potential benefits of renewable energy, have enabled the deployment of solar 
PV storage facilities fully integrated into a utility power grid. An example of such a facility is the Prosperity Energy Storage Project managed by the Public Service Company of New Mexico (PNM) in New Mexico, the first US solar PV storage facility fully integrated into a utility's power grid [2]. One of the primary functions of the battery storage at the Prosperity Site is to solve the problem of intermittency in the solar PV production due to rapid variations in irradiance. However, the batteries are also used to shift peak PV production to be in phase with peak demand.

Multiple factors need to be investigated to determine how energy should be distributed from difference sources. In the traditional model, electric power is delivered unidirectionally from a conventional power plant to the distribution substation to the customer. However, with the advent of alternative sources of energy and advances in energy storage technologies, a challenge and opportunity presents itself in determining how to optimally schedule the energy generated from difference sources to satisfy total demand.

This paper addresses the optimization problem where coupled photovoltaic, battery storage and conventional sources of energy are scheduled to satisfy a certain energy demand and maximize profit. The paper models the scheduling problem as a Linear Program (LP) with a finite number of constraints and variables. Using numerical examples, the effectiveness of the proposed LP is demonstrated.

The paper is organized as follows. Section 2 discusses related work. Section 3 provides an overview of the system and presents terminology. Section 4 presents the mathematical model and its complexity. Section 5 shows numerical results, and Section 6 concludes.

\section{Related Work}

Many authors have addressed optimization issues in the context of renewable energy. Hill [3] proposes a solution for an optimization problem that focuses on minimizing the costs paid by a specific customer when meeting demand using conventional and solar energy. Hourly increments of time for a whole week are used to minimize cost. Power supplied by the solar energy source and battery are assumed free. Limits on charging and discharging rates are enforced. Switching the battery operation from a charging state to discharging state and back are assumed detrimental to battery life and are tracked in the algorithm. Nottrott et al. [4] minimize the amount of energy purchased by a customer from the electrical grid when the demand 
can be met by the electrical grid, solar and battery storage. The customer can also sell energy to the grid. They find that the net value of the battery increased when operated on the optimized schedule. Riffonneau [1] minimizes the final value of cash flow using a power scheduling algorithm based on dynamic programming in a coupled system. The solar generator, batteries and converter are modeled based on parameters which include temperature, battery aging and power input. Lu [5] minimizes the cost of conventional sources of energy in a combined conventional/PV/battery storage system. Multiple conventional thermal sources are used and the start-up and shutdown costs of the thermal units are included in their optimization. The optimization is performed with a Lagrangian relaxation-based optimization algorithm. Zhang et al. [6] minimize the total cost of a hybrid system which potentially incorporates wind, photovoltaic and battery sources. Marwali et al. [7] use Lagrangian relaxation and dynamic programming to minimize a function of the conventional (thermal) contribution to the grid. Many effects are accounted for including battery and photovoltaic capacity as well as ramp rates for conventional thermal contributions. Teng [8] uses a genetic algorithm to optimize charging and discharging of battery storage systems to minimize line losses incurred during stages of the charging process. Abbaspour et al. [9] use a mixed integer non-linear programming formulation to optimize profits from the operation of a conventional gas generator coupled with wind power and compressed air energy storage. Vijay et al. [10] use convex optimization to maximize the production of clean water in a plant powered by solar power with battery reserves.

Our approach takes the perspective of the utility company and optimizes profit. The algorithm is computationally fast and can produce scheduling projections under a minute. In addition, the algorithm is general enough so that it can run over multiple days using hours or fractions of an hour. Convergence is demonstrated when the increments of time are decreased.

\section{Terminology}

This paper presents a strategy to maximize profit by determining the optimal scheduling of a coupled photovoltaic, battery storage and conventional system. The proposed scheme can optimize scheduling for increments of time ranging from minutes to hours. Figure 1 illustrates the system. Assuming two different sources of energy, conventional and solar, the terminology in 
Table 1 will be used in the rest of the paper. The unit of energy is the kilowatt-hour (kWh).

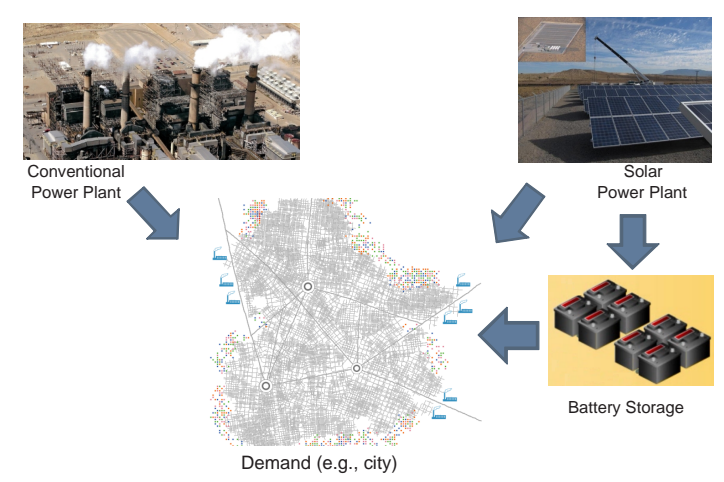

Figure 1: Illustration of the energy scheduling problem with two sources of energy and battery storage that need to satisfy an energy demand.

The model includes a battery bank that can store energy from the solar (PV) source, energy that can subsequently be dispatched at a later time. The battery has an initial amount of energy $B_{\text {start }}$, and lower and upper capacity bounds $B_{\min }$ and $B_{\max }$. The operational costs required to produce and deliver energy from the conventional, solar, and battery sources in hour $i$ are quantified by $C_{i}^{C}, C_{i}^{P V}$ and $C_{i}^{B}$. The energy demand to be satisfied in hour $i$ is given by $D_{i}$. Note that this demand varies during the day and increases during the afternoon in typical cities. Our model currently assumes that the conventional energy source cannot recharge the battery storage.

The solar plant or solar source generates $E_{i}^{P V_{\text {prod }}} \mathrm{kWh}$ in hour $i$. No limitation is imposed on the conventional power plant production, since real-world scenarios show that the proportion of energy produced from solar sources is much lower than conventional energy sources. Note that the amount of energy generated by the solar plant depends on the time the energy is generated; e.g., at noon time $t=12$, the energy $E_{12}^{P V_{\text {prod }}}$ is greater than the energy generated in the early morning, $t=6, E_{6}^{P V_{\text {prod }}}$.

Batteries can be recharged by energy generated by the solar plant at a maximum rate of $E_{\text {Charge }}^{P V B} \mathrm{kWh}$ per hour; similarly, the battery can subsequently release at most $E_{\text {Discharge }}^{B} \mathrm{kWh}$ per hour.

The cost for a user per $\mathrm{kWh}$ in hour $i$ is $H_{i}$ (in $\$ / \mathrm{kWh}$ ). The profit for the energy company per unit of energy from the conventional, solar, and battery sources is given by $P_{i}^{C}, P_{i}^{P V}$, and $P_{i}^{B}$ respectively. Note that the profit is 


\begin{tabular}{|c|c|}
\hline Notation & Meaning \\
\hline$B_{\text {start }}$ & Initial energy stored by battery $(\mathrm{kWh})$ \\
\hline$B_{i}$ & Battery energy at time interval $i(\mathrm{kWh})$ \\
\hline$B_{\min }$ & Minimum battery storage allowed (kWh) (imposed to avoid deep cycling) \\
\hline$B_{\max }$ & Maximum battery storage allowed (kWh) \\
\hline$C_{i}^{C}$ & Cost to supply a $\mathrm{kWh}$ by the conventional power plant \\
\hline$C_{i}^{P V}$ & Cost to supply a $\mathrm{kWh}$ by the solar power plant \\
\hline$C_{i}^{B}$ & Cost to supply a kWh by battery storage to grid \\
\hline$D_{i}$ & Energy $(\mathrm{kWh})$ demand \\
\hline$E_{i}^{P V_{\text {prod }}}$ & Energy $(\mathrm{kWh})$ produced by the solar power plant \\
\hline$E_{\text {Discharge }}^{B}$ & Maximum discharge energy $(\mathrm{kWh})$ in one hour from battery storage \\
\hline$E_{\text {Charge }}^{P V B}$ & Maximum energy $(\mathrm{kWh})$ that can be used to charge battery storage \\
\hline$E_{i}^{C}$ & Energy $(\mathrm{kWh})$ discharged by the conventional power plant to satisfy demand \\
\hline$E_{i}^{P V}$ & Energy (kWh) discharged by the solar power plant to satisfy demand \\
\hline$E_{i}^{B}$ & Energy (kWh) discharged by the battery storage to grid \\
\hline$E_{i}^{P V B}$ & Energy (kWh) used by the solar power plant to charge battery storage \\
\hline$H_{i}$ & Charge $(\$ / \mathrm{kWh})$ by electrical utility company to customers \\
\hline$P_{i}^{C}$ & Profit per energy unit $(\$ / \mathrm{kWh})$ from the conventional plant \\
\hline$P_{i}^{P} P V$ & Profit per energy unit $(\$ / \mathrm{kWh})$ from the solar plant \\
\hline$P_{i}^{B}$ & Profit per energy unit $(\$ / \mathrm{kWh})$ from the battery storage \\
\hline
\end{tabular}

Table 1: Notation used to model the power scheduling problem. The subscript $i$ refers to hour $i$.

also indexed by the hour $i$ in which the service is delivered.

The variables $E_{i}^{C}, E_{i}^{P V}, E_{i}^{B}$ quantify the amount of energy discharged to satisfy the demand at time $i$ from the conventional, solar, and battery sources. We use the variable $E_{i}^{P V B}$ to indicate the amount of energy diverted to recharge the battery in hour $i$ from the PV source.

\section{Mathematical Model}

\subsection{Mathematical Formulation}

The proposed model considers the variation on energy demand from hour to hour. Our objective is to maximize the total profit over a day $P$ :

$$
P=\sum_{i=1}^{24}\left[P_{i}^{C} E_{i}^{C}+P_{i}^{P V} E_{i}^{P V}+P_{i}^{B} E_{i}^{B}\right] .
$$


The function $P$ computes the total profit for one day using hourly increments. While the proposed model has been generalized to maximize profit over multiple days using any fraction of an hour (e.g., 1, 5, 10, 15, 20, 30 minutes), our formulation here uses hourly increments of time for simplicity. The profit function is subject to the following constraints.

The different sources of energy must satisfy the energy demand $D_{i}$ :

$$
E_{i}^{C}+E_{i}^{P V}+E_{i}^{B}=D_{i} .
$$

Using Eq. (2), the objective function $F$ can be rewritten as

$$
P=\sum_{i=1}^{24}\left[P_{i}^{C}\left(D_{i}-E_{i}^{P V}-E_{i}^{B}\right)+P_{i}^{P V} E_{i}^{P V}+P_{i}^{B} E_{i}^{B}\right] .
$$

The model also considers the fact that solar energy can be used to charge the battery. Thus the solar plant contribution to the grid plus the solar plant contribution to battery storage $E_{i}^{P V B}$ in hour $i$ needs to be less or equal to the solar plant production $E_{i}^{P V_{\text {prod }}}$

$$
E_{i}^{P V}+E_{i}^{P V B} \leq E_{i}^{P V_{\text {prod }}},
$$

for $i=1,2, \ldots 24$. Since there are 24 hours in a day, there are $24 E_{i}^{P V}, 24$ $E_{i}^{P V B}$ and $24 E_{i}^{B}$ variables for a total of 72 variables. tive,

The conventional power plant contribution to the grid must be nonnega-

$$
\left(E_{i}^{C}=D_{i}-E_{i}^{P V}-E_{i}^{B}\right) \geq 0
$$

for $i=1,2, \ldots 24$. The amount of energy stored in the battery at the end of hour $i$ cannot exceed the maximum battery charge $B_{\text {max }}$,

$$
B_{i}=B_{\text {start }}+\sum_{j=1}^{i}\left(E_{j}^{P V B}-E_{j}^{B}\right) \leq B_{\max }
$$

for $i=1,2, \ldots 24$. The battery charge at the end of hour $i$ needs to be greater than $B_{\min }$ to avoid deep cycle discharge,

$$
B_{i}=B_{\text {start }}+\sum_{j=1}^{i}\left(E_{j}^{P V B}-E_{j}^{B}\right) \geq B_{\min }
$$


for $i=1,2, \ldots 24$. Finally, the battery needs to be returned to a fraction $\eta$ of its original state of charge for the next cycle,

$$
B_{24}=B_{\text {start }}+\sum_{j=1}^{24}\left(E_{j}^{P V B}-E_{j}^{B}\right) \geq \eta B_{\text {start }} .
$$

The parameter $\eta$ is set to 1 for the numerical examples presented in this paper. Thus, the battery must be recharged to a level of charge greater or equal to its initial state at the end of the scheduling period. Additionally, the maximum rate at which the battery storage can be charged or discharged is

$$
\begin{aligned}
& E_{i}^{P V B} \leq E_{\text {Charge }}^{P V B}, i=1,2, \ldots, 24 \\
& E_{i}^{B} \leq E_{\text {Discharge }}^{B}, i=1,2, \ldots, 24 .
\end{aligned}
$$

Equation (10) can be reformulated as

$$
E_{i}^{B} \leq E_{\text {Discharge }}^{B}\left[1-\alpha+\alpha\left(\frac{B_{i}-B_{\min }}{B_{\max }-B_{\min }}\right)\right], i=1,2, \ldots, 24 .
$$

to allow for a discharge rate that is dependent on the state of charge. If $B_{i}=B_{\max }$, the discharge energy will be maximized. However, if $B_{i}=B_{\min }$, the discharge energy will be reduced by a factor $\alpha$.

Figure 2 illustrates the complete LP problem including the objective function and the constraints discussed above. In equation (17),

$$
\tilde{E}=E_{\text {Discharge }}^{B}(1-\alpha)+\alpha E_{\text {Discharge }}^{B}\left(\frac{B_{\text {start }}-B_{\min }}{B_{\max }-B_{\min }}\right) .
$$

For our calculations, we set $\alpha=0$. Note that the objective function and constraints are linear functions of the variables $E_{i}^{B}, E_{i}^{P V}$, and $E_{i}^{P V B}$. Thus, the model can be solved by an LP algorithm.

\subsection{Complexity}

Let $n$ be the number of variables of the LP shown in Figure 2. According to Equation (20), the proposed model has $n=24 \cdot 3=72$ variables. Similarly, let $m$ be the number of constraints of the LP. Equations (13)-(18) each have 24 constraints. Equation (19) represents one additional constraint. We do not count (20) as a constraint because the non-negativity of the variables is an assumption built into algorithm we use. Thus, the total number of 


$$
\begin{array}{rlrl}
\operatorname{Max} F=\sum_{i=1}^{24}\left(P_{i}^{P V}-P_{i}^{C}\right) E_{i}^{P V} & +\left(P_{i}^{B}-P_{i}^{C}\right) E_{i}^{B}+P_{i}^{C} D_{i} \\
E_{i}^{P V}+E_{i}^{P V B} & \leq E_{i}^{P V \text { prod }} & i=1,2, \ldots, 24 \\
E_{i}^{P V}+E_{i}^{B} & \leq D_{i} & i=1,2, \ldots, 24 \\
\sum_{j=1}^{i}\left(E_{j}^{P V B}-E_{j}^{B}\right) & \leq B_{\text {max }}-B_{\text {start }} & i=1,2, \ldots, 24 \\
\sum_{j=1}^{i}\left(E_{j}^{B}-E_{j}^{P V B}\right) & \leq B_{\text {start }}-B_{\text {min }} & i=1,2, \ldots, 24 \\
E_{i}^{B}+\frac{}{\alpha E_{\text {Discharge }}^{B}}\left(B_{\text {max }}-B_{\text {min }}\right) \sum_{j=1}^{i}\left(E_{j}^{B}-E_{j}^{P V B}\right. & \leq \tilde{E}^{2} & \\
\sum_{j=1}^{24}\left(E_{j}^{B}-E_{j}^{P V B}\right) & \leq(1-\eta) B_{\text {start }} & \\
E_{i}^{P V}, E_{i}^{B}, E_{i}^{P V B} & \geq 0 & i=1,2, \ldots, 24,24
\end{array}
$$

Figure 2: Linear program for the power generation optimization problem.

constrains is $m=24 \cdot 6+1=145$. In general, $n=k \cdot 3$ and $m=k \cdot 6+1$, where $k$ is a parameter representing the number of intervals per day (e.g., $k=24$ for intervals of one hour).

For a finer granularity, $k$ can be adjusted so that the time intervals represent fractions of hours. For practical purposes, $k$ would not be a very large number; for example, a granularity of a minute, which would already be very granular, would result in $k=24 \cdot 60=1440$.

For daily (24 hours) or even weekly schedules, the number of variables $n$ and the number of constraints $m$ are bounded. The LP of Figure 2 can then be solved in a reasonable time by any polynomial time algorithm such as interior point methods. The time complexity of such methods applied to the LP of Figure 2 will be $O\left(n^{2} m\right)$ [11]. The simplex method is used to solve the optimization problem in the numerical simulations presented in this paper 
using an adaption of the code provided by Moreau [12]. While simplex is an exponential-time algorithm, exponential examples do not occur in practice.

\section{Numerical Examples}

This section presents a numerical example of how the LP of Figure 2 is used to solve variations of the scheduling problem. Assume a town has an energy demand in kWh as shown in Figure 3. A total of 207,375 kWh are needed to be produced to satisfy the demand during the day. Note that the time period of maximum energy demand is between $6 \mathrm{PM}$ and $7 \mathrm{PM}$, and that energy demand between 4 PM to 9 PM is greater than that between other time periods. This demand profile represents a typical scenario where energy is consumed in greater quantities by households once they return home after a work day.

Profits $P_{i}^{C}, P_{i}^{P V}$, and $P_{i}^{B}$ are calculated according to the following equations:

$$
\begin{aligned}
P_{i}^{C} & =H_{i}-C_{i}^{C}, \\
P_{i}^{P V} & =H_{i}-C_{i}^{P V}, \\
P_{i}^{B} & =H_{i}-C_{i}^{B} .
\end{aligned}
$$

Recall that $H_{i}$ is the charge per energy unit (kWh), and $C_{i}^{C}, C_{i}^{P V}$, and $C_{i}^{B}$ are the supply costs per energy unit for the conventional, solar, and battery sources at time interval $i$. Equations (21), (22), and (23) represent the net profit of supplying energy from the respective sources.

The numerical examples assume the following charge and cost rates in dollars per kWh,

$$
\begin{aligned}
H_{i} & =.1150 \\
C_{i}^{C} & =\max \left\{.08, .10 \frac{D_{i}}{\max _{i}\left\{D_{i}\right\}}\right\}, \\
C_{i}^{P V} & =.01 \\
C_{i}^{B} & =.011 .
\end{aligned}
$$

Equation (25) indicates that $C_{i}^{C}$, the cost per kWh of supplying energy from the conventional source, is constant if the demand at time $i$ is less than the $80 \%$ of the peak demand; otherwise, the cost increases linearly with the demand. This incentivizes the use of nonconventional energy sources 
during peak demand hours. This policy may reflect the fact that the energy company is approaching its generation limit and may need extra resources to increase production. For example, in New Mexico, a residential service is charged at a lower rate for the first $450 \mathrm{kWh}$ [13]. Ideally $C_{i}^{C}$ should be a function of $E_{i}^{C}$, the energy supplied by the conventional source. However, such a functional dependence would make the problem nonlinear and for this reason, we retain the functional dependence of $C_{i}^{C}$ on $D_{i}$. Equation (26) indicates that the cost of supplying energy from the solar source is lower than that of conventional sources; this reflects the fact that, once deployed, solar energy is cheaper than that of conventional sources. However, initial construction and installation costs are not accounted for in this equation. Equation (27) shows that the cost of supplying energy from the battery is slightly higher than that from the solar source (26); this higher cost accounts for current and storage losses.

The following maximum, minimum and starting charges in $\mathrm{kWh}$ for the battery storage are assumed in the numerical examples:

$$
\begin{gathered}
B_{\max }=20000 \\
B_{\min }=.3 B_{\max }, \quad B_{\text {start }}=.7 B_{\max } .
\end{gathered}
$$

Finally, the following maximum energy charge and discharge amounts in one hour are assumed:

$$
\begin{gathered}
E_{\text {Charge }}^{P V B}=2000 \mathrm{kWh}, \\
E_{\text {Discharge }}^{B}=4000 \mathrm{kWh} .
\end{gathered}
$$

The above values may be adjusted to a particular battery technology.

The demand $D_{i}$ and solar energy output $E_{i}^{P V_{\text {prod }}}$ are plotted in Figure 3. The peak demand is $10,000 \mathrm{kWh}$ in hour 19 (6 PM-7 PM). The peak photovoltaic power output (3000 kWh) occurs in hour 14. The photovoltaic output is computed based on the direct irradiance of the sun which is based on the day of the year, the hour of the day, the latitude of the location and the angle at which the photovoltaic array is tilted. The relation between irradiance and solar energy output is given by the following equation:

$$
E_{i}^{P V_{\text {prod }}}=\xi I A
$$

where $I$ represents the average irradiance, $A$ the area of the solar array, and $\xi$ the efficiency which is primarily a function of panel temperature. The irradiance is computed using the cosine of the angle between the panel normal and 


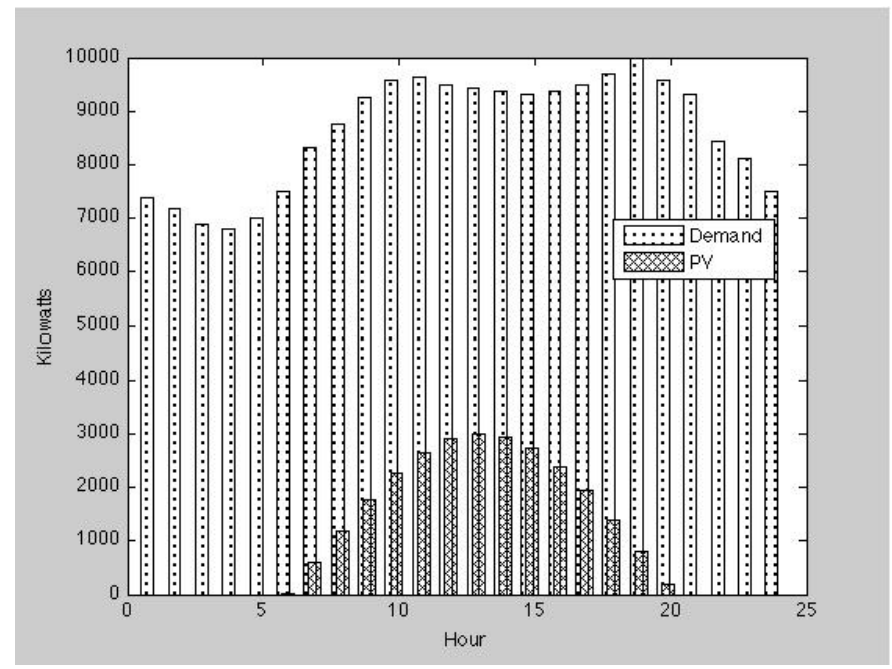

Figure 3: Demand and solar output (in kilowatt-hours) as a function of hour.

the sun's incident rays. We developed a code based on equations explained in $[14]$.

Figure 4 shows the result of a 24 hour simulation when 1 hour increments of time are used. The y-axis represents the energy contributions from the blue (conventional), light blue (PV source), and battery (brown). In addition, the yellow bar represents the energy from the PV source used to recharge the battery. The blue, light blue and brown bars must sum up to be the total demand in hour $i$ as shown in Figure 3. For example, at time $i=10$, the total production is $9,563 \mathrm{kWh}$; all three energy sources contribute to satisfy the immediate demand. On the other hand, at time $i=8$, the conventional source is used exclusively to satisfy the demand while PV production is used to recharge the battery. Note that the solar source charges the battery storage (yellow) in non-peak demand hours so the battery storage can discharge energy in peak demand hours (brown). This solution is consistent with the fact that, according to Equation (25), the energy from the conventional source is more expensive at peak hours. The optimized schedule of Figure 4 thus avoids the overutilization of the conventional source at peak hours by releasing energy from the battery source, which is charged at nonpeak hours. Similar results, shown in Figure 5, are obtained for finer time interval resolutions of 30 minutes and 15 minutes. Note that the scheduling results do not differ significantly from those obtained with time intervals of 


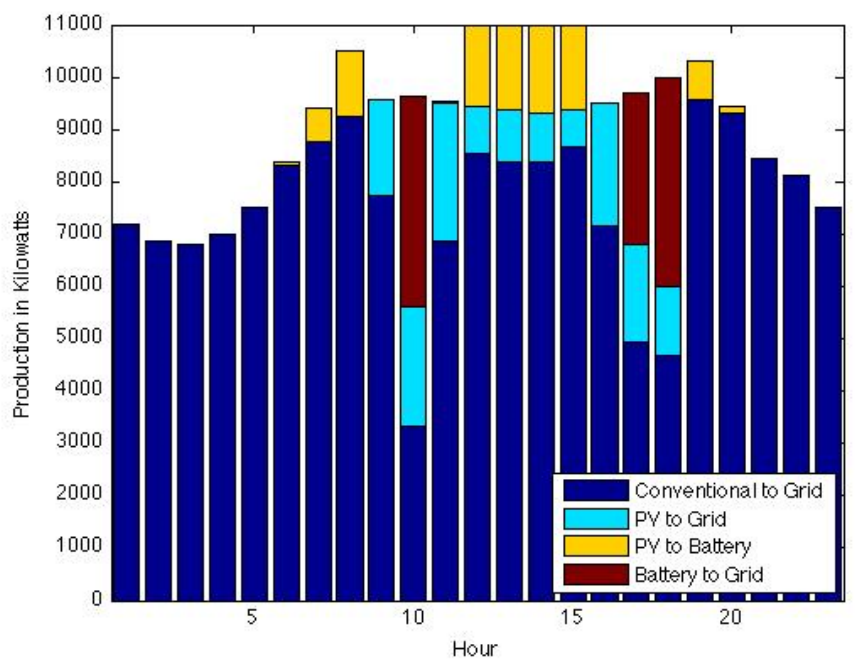

Figure 4: Optimal scheduling of coupled conventional, photovoltaic, battery and photovoltaic charging in a 24 hour day using 1 hour time intervals.

1 hour (Figure 4). For this particular cost configuration, we note that the optimal profit is only slightly higher than the profit obtained without the use of batteries: $.49 \%$ for the hourly interval, $.53 \%$ for the 30 minute interval and $.55 \%$ for the 15 minute interval. However, a true measure of profit would require the conventional cost $C_{i}^{C}$ to be function of $E_{i}^{C}$. Cost penalties for decreasing or increasing conventional energy production would need to be included in Equation (25).

Figure 6 shows the results of simulations performed over 2 and 3 days respectively when 15 minute intervals of time are used. The optimal schedule to satisfy a two-day demand (Figure 6, left) shows similar daily patterns: the solar source charges the battery storage (yellow) in non-peak demand hours so the battery storage can discharge energy in peak demand hours (brown). There are slight differences from day one (hours 1-24) to day two (hours 25-48); in particular, the amount of energy from the battery delivered to satisfy the demand (battery to grid) slightly decreases on day two. On the other hand, the energy from solar sources used to recharge the battery increases. This is due to the fact that the battery needs to be recharged to its initial state. Similar conclusions can be derived from the results in the three-day simulation shown in Figure 6 (right). The energy schedule shows 

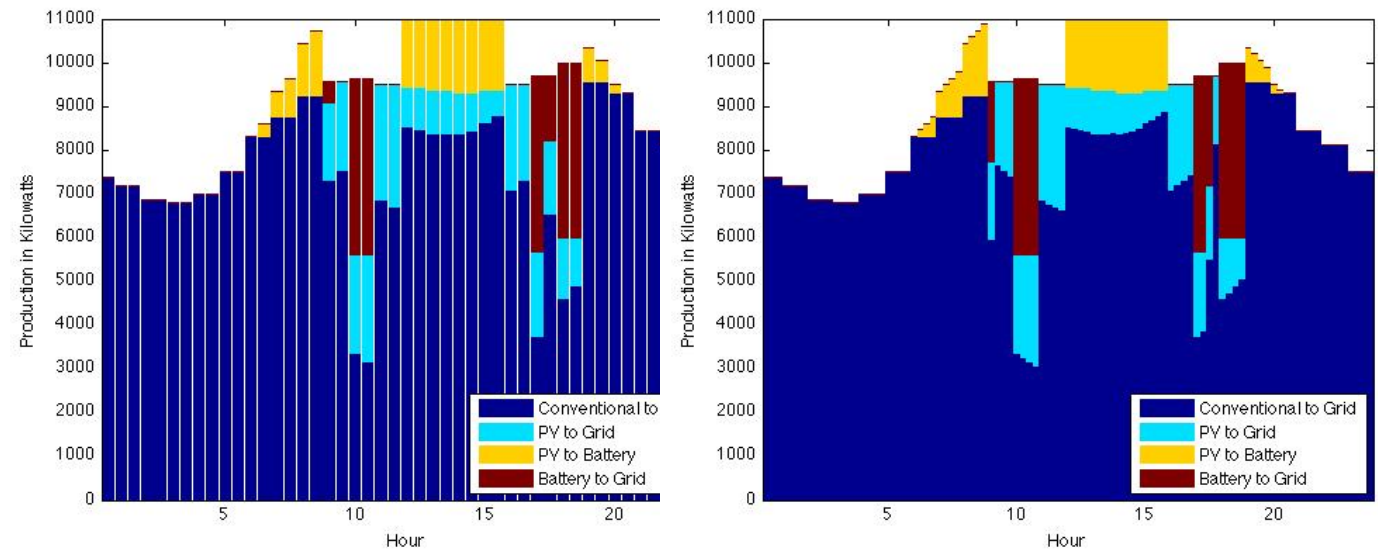

Figure 5: Optimized distribution of conventional, photovoltaic, battery and photovoltaic charging in 24 hour day using 30 minute intervals (left) and 15 minute intervals (right).
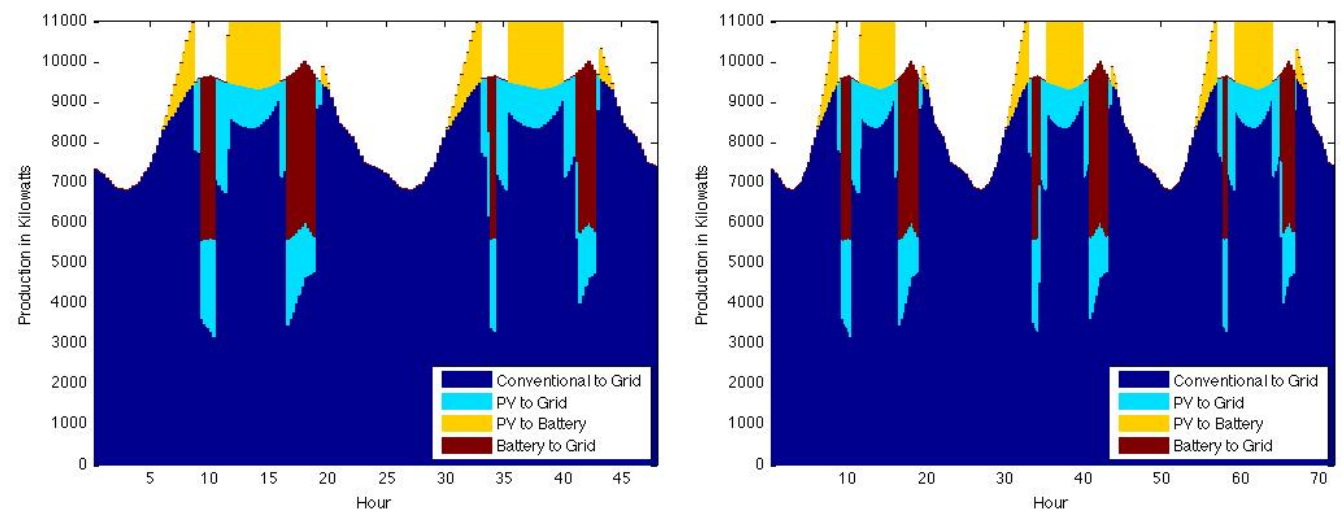

Figure 6: Optimized distribution of conventional, photovoltaic, battery and photovoltaic charging over 48 (left) and 72 (right) hours using 15 minute intervals. 
Table 2: Running time of difference instances of LP presented in the paper and solved with a MATLAB implementation of the simplex algorithm.

\begin{tabular}{ccc}
\hline \hline Length of time & Time interval & Time \\
\hline 24 hours & 1 hour & $2.4 \mathrm{~s}$ \\
24 hours & .5 hour & $2.5 \mathrm{~s}$ \\
24 hours & .25 hour & $3.8 \mathrm{~s}$ \\
24 hours & .125 hour & $16 \mathrm{~s}$ \\
48 hours & .25 hour & $16 \mathrm{~s}$ \\
72 hours & .25 hour & $52 \mathrm{~s}$ \\
\hline
\end{tabular}

a daily pattern again. This scheduling pattern can be applied to regions where the solar energy does not vary substantially over a series of cloudless days. An example of this is New Mexico, a state with more than 300 days of sunshine per year. On the other hand, if the amount of energy from the solar source varies, the schedule produced by the LP of Figure 2 would be adjusted accordingly.

Actual power production from a PV site can vary significantly over the course of a day. Figure 7 shows actual smoothed hourly power production from the PNM Prosperity Site from June 9, 10 and 11 in 2012. Figure 8 shows the optimized distribution of conventional, photovoltaic and battery contributions to the grid. In the simulation, we assume the same profile as Figure 3 with a peak demand of $20,000 \mathrm{~kW}$ which is representative of the Albuquerque region. The charge $E_{\text {Charge }}^{P V B}$ and discharge rate $E_{\text {Discharge }}^{B}$ are assumed to be $500 \mathrm{~kW}$. The battery capacity $B_{\max }$ is assumed to be 1000 $\mathrm{kWh}$.

Table 2 shows the running time for the different instances of the LP of Figure 2. The LP was solved with an implementation of the simplex algorithm in MATLAB which ran on a Laptop Dell Inspiron computer with $6 \mathrm{~GB}$ of RAM and a $2.3 \mathrm{GHz} \mathrm{CPU}$ clock. Note that the computational times listed in Table 2 is for reference purposes, to demonstrate that the different instances are solved in polynomial time. In compiled programming languages, the running time would be much faster.

\section{Conclusions}

This paper presents an optimization model for scheduling coupled photovoltaic, battery and conventional energy sources to satisfy energy demands 


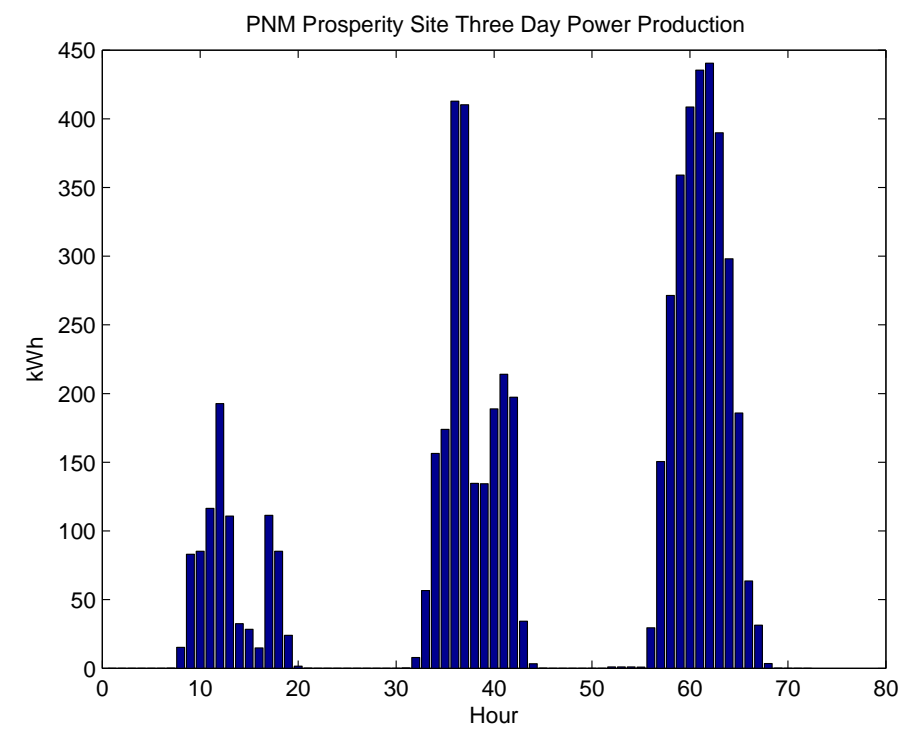

Figure 7: Actual three day power production from PNM prosperity site.

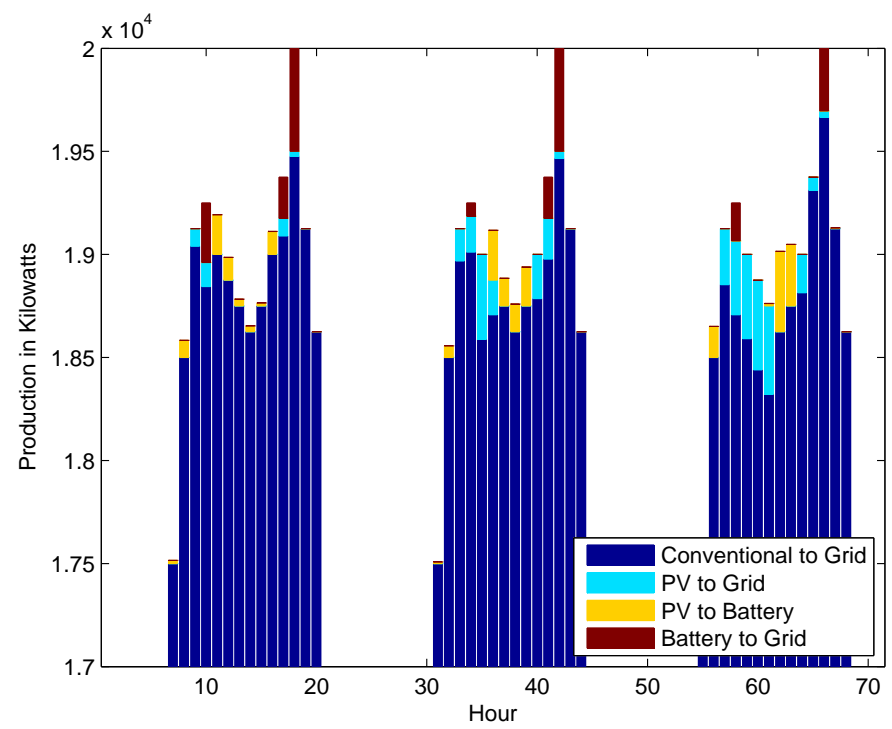

Figure 8: Optimized distribution of conventional, photovoltaic, battery and photovoltaic energy based on three day PNM power production. 
over multiple days and arbitrary increments of time. The optimization scheme maximizes profit and is modeled as a fast adaptable linear program and can be solved in polynomial time. The model thus allows the optimal use of resources from a coupled photovoltaic, battery storage and conventional power system. This optimal combination of resources allows utilities to effectively utilize the new technologies currently being deployed across the country, in particular new energy storage systems that have been a barrier to realizing the benefits of solar energy [2].

\section{Acknowledgement}

The authors would like to acknowledge the PNM support of this research through a grant award from the Department of Energy under Award Number DE-OE0000230.

\section{References}

[1] Riffonneau Y, Bacha S. Optimal power flow management for grid connected PV systems with batteries. IEEE Transactions on Sustainable Energy 2011;2;309-320.

[2] PNM Prosperity Energy Storage Project, https://share.pnmresources.com/Public/Pages/default.aspx, September 2013 .

[3] Hill J, Nwankpa C. System constraints effects on optimal dispatch schedule for battery storage systems. ISGT '12 Proceedings of the 2012 IEEE PES Innovative Smart Grid Technologies 2012;1-8.

[4] Nottrott A, Kleissl J, Washom B, Energy dispatch schedule optimization and cost benefit analysis for grid-connected, photovoltaic-battery storage systems. Renewable Energy. 2013;55;230-240.

[5] Lu B, Shahidehpour M., Short-term scheduling of battery in a gridconnected PV/battery system. IEEE Transactions on Power Systems. 2005;20;1053-1061.

[6] Zhang L, Barakat G, Yassine A. Design and optimal sizing of hybrid $\mathrm{PV} /$ wind/diesel system with battery storage by using DIRECT search 
algorithm. 15th International Power Electronics and Motion Control Conference (EPE-PEMC) Novi Sad, Serbia 2012.

[7] Marwali M, Haili M, Shahidehpour S, Abdul-Rahman K, Short term generation scheduling in photovoltaic-utility grid with battery storage. IEEE Transactions on Power Systems 1998;13;1057-1062.

[8] Teng J, Luan S, Lee D, Huang Y. Optimal charging/discharging scheduling of battery storage systems for distribution systems interconnected with Sizeable PV generation systems. IEEE Transactions on Power Systems 2013;28;1425-1433.

[9] Abbaspour M, Satkin M, Mohammadi-Ivatloo B, Hoseinzadeh Lotfi F, Noorollahi Y. Optimal operation scheduling of wind power integrated with compressed air energy storage (CAES). Renewable Energy 2013;51;53-59.

[10] Vijay A, Ling KV, Fane AG, Reserve management and real time optimization for a solar powered Membrane Distillation Bio-Reactor water recycling plant via convex optimization. Renewable Energy 2013;60;489497.

[11] Boyd S, Vandenberghe L. Convex Optimization. Cambridge University Press; 2004.

[12] Moreau, Jean-Pierre, // http://jean-pierre.moreau.pagespersoorange.fr/index.html, September 2013.

[13] Electricity rates, Public Service Company of New Mexico, http://www.pnm.com/regulatory/_legacy.htm, September 2013.

[14] Stine, W.B. and Michael Geyer, Power from the Sun, www.powerfromthesun.net/book.html, Stine and Geyer, 2001, Chapters 3 and 4. 